\title{
New Probability Distributions in Astrophysics: VI. The Truncated Sujatha Distribution
}

\author{
Lorenzo Zaninetti \\ Physics Department, Turin, Italy \\ Email:1.zaninetti@alice.it
}

How to cite this paper: Zaninetti, L. (2021) New Probability Distributions in Astrophysics: VI. The Truncated Sujatha Distribution. International Journal of Astronomy and Astrophysics, 11, 517-529. https://doi.org/10.4236/ijaa.2021.114028

Received: October 28, 2021

Accepted: December 21, 2021

Published: December 24, 2021

Copyright $\odot 2021$ by author(s) and Scientific Research Publishing Inc. This work is licensed under the Creative Commons Attribution International License (CC BY 4.0).

http://creativecommons.org/licenses/by/4.0/

\section{(c) (i) Open Access}

\begin{abstract}
The truncated version of the two-parameter Sujatha distribution is analysed. In particular, its probability density function and distribution function are obtained. The results are applied to the initial mass function for stars, to the luminosity function for galaxies, to the number of galaxies as a function of the redshift and to the average absolute magnitude of a galaxy as a function of its redshift.
\end{abstract}

\section{Keywords}

Stars: Mass Function, Galaxy Groups, Clusters, Superclusters, Large Scale Structure of the Universe, Cosmology

\section{Introduction}

The one-parameter Sujatha distribution was introduced by [1], deriving the most important statistical quantities This distribution has been generalized in various ways [2] [3] [4] [5] [6]. Some questions are still to be analysed:

1) Is it possible to introduce the effect of truncation for the Sujatha distribution?

2) Is the Sujatha distribution useful to model the initial mass function (IMF) for the stars?

3) Is it possible to model the luminosity function for galaxies (LF) with the Sujatha distribution?

This paper reviews the Sujatha distribution in Section 2, introduces the scale in Section 3, evaluates the main statistical parameters of left- and right-truncated Sujatha distributions with scale in Section 4, derives the luminosity function (LF) for galaxies in Section 5, derives the truncated LF for galaxies in 6 and applies the results to same astrophysical samples, see Section 7. 


\section{The Sujatha Distribution}

Let $X$ be a random variable with values in $[0, \infty]$. Then, the one-parameter $S u$ jatha probability density function (PDF), $f(x)$, is

$$
f(x ; b)=\frac{b^{3}\left(x^{2}+x+1\right) \mathrm{e}^{-b x}}{b^{2}+b+2},
$$

where $b$ is the shape parameter, see [1]. Figure 1 shows a 3D surface of this PDF.

The distribution function (DF), $F(x)$, is

$$
F(x ; b)=\frac{\left(-2+\left(-x^{2}-x-1\right) b^{2}+(-2 x-1) b\right) \mathrm{e}^{-b x}+b^{2}+b+2}{b^{2}+b+2} .
$$

Its average value or mean, $\mu$, is

$$
\mu(b)=\frac{b^{2}+2 b+6}{b\left(b^{2}+b+2\right)},
$$

its variance, $\sigma^{2}$, is

$$
\sigma^{2}(b)=\frac{b^{4}+4 b^{3}+18 b^{2}+12 b+12}{b^{2}\left(b^{2}+b+2\right)^{2}},
$$

its skewness is

$$
\operatorname{skewness}(b)=\frac{2 b^{6}+12 b^{5}+72 b^{4}+88 b^{3}+108 b^{2}+72 b+48}{\left(b^{4}+4 b^{3}+18 b^{2}+12 b+12\right)^{\frac{3}{2}}},
$$

and its kurtosis is

kurtosis $(b)=\frac{9 b^{8}+72 b^{7}+516 b^{6}+1128 b^{5}+2208 b^{4}+2592 b^{3}+2736 b^{2}+1440 b+720}{\left(b^{4}+4 b^{3}+18 b^{2}+12 b+12\right)^{2}}$.

Its $k$ th moment about the origin, $\mu_{k}^{\prime}$, is

$$
\mu_{k}^{\prime}(b)=\frac{b^{-k}(k+2) !+b^{1-k}(1+k) !+b^{-k+2} k !}{b^{2}+b+2} .
$$

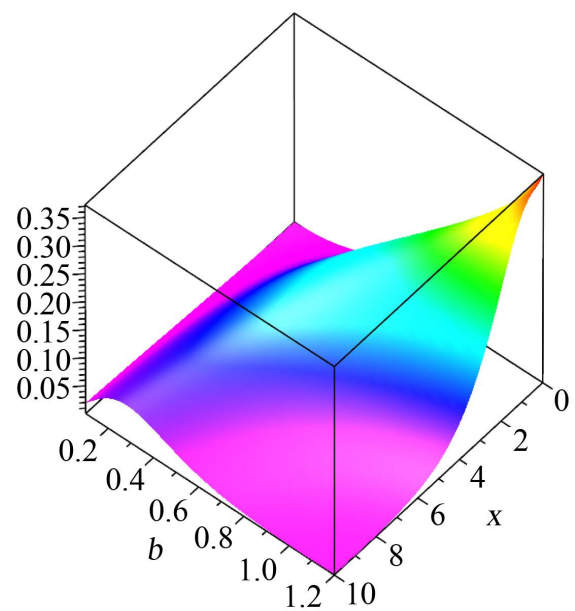

Figure 1. The Sujatha PDF as a function of the parameter $b$ and the random variable. 
Its mode, $\operatorname{Mode}(b)$, is

$$
\operatorname{Mode}(b)=\frac{-b+2+\sqrt{-3 b^{2}+4}}{2 b} .
$$

An approximate expression for its median, $\operatorname{Med}(b)$, can be obtained by a Taylor expansion of order 2 for the DF around the average value

$$
\operatorname{Med}(b)=\frac{-\left(b^{2}+b+2\right)^{4} \mathrm{e}^{\frac{b^{2}+2 b+6}{b^{2}+b+2}}+4 b^{8}+20 b^{7}+84 b^{6}+220 b^{5}+494 b^{4}+804 b^{3}+1104 b^{2}+984 b+704}{2 b\left(b^{2}+b+2\right)\left(b^{2}+3 b+3\right)\left(b^{4}+6 b^{2}+12\right)} \text {. }
$$

As an example, the above approximate median at $b=1$ has a percentage error of $1.11 \%$. The moment generating function, $M_{X}$, is

$$
M_{X}(t ; b)=\frac{\left(b^{2}-2 b t+t^{2}+b-t+2\right) b^{3}}{\left(b^{2}+b+2\right)(b-t)^{3}} .
$$

A random generation of the Sujatha variate $X$ can be obtained by solving the nonlinear equation

$$
F(x ; b)=R,
$$

where $R$ is the unit rectangular variate and $F$ is given by Equation (2). The parameter $b$ can be derived from the sample mean, $\bar{x}$, by solving the cubic Equation (3)

$$
\begin{aligned}
& b=\frac{\left(3 \sqrt{3} \sqrt{7 \bar{x}^{4}+28 \bar{x}^{3}+171 \bar{x}^{2}+32 \bar{x}+5} \bar{x}+8 \bar{x}^{3}+66 \bar{x}^{2}+6 \bar{x}+1\right)^{\frac{1}{3}}}{3 \bar{x}} \\
& -\frac{(\bar{x}-1)(5 \bar{x}+1)}{3 \bar{x}\left(3 \sqrt{3} \sqrt{7 \bar{x}^{4}+28 \bar{x}^{3}+171 \bar{x}^{2}+32 \bar{x}+5} \bar{x}+8 \bar{x}^{3}+66 \bar{x}^{2}+6 \bar{x}+1\right)^{\frac{1}{3}}}-\frac{\bar{x}-1}{3 \bar{x}} .
\end{aligned}
$$

\section{The Sujatha Distribution with Scale}

The two-parameter Sujatha PDF, $f_{s}(x)$, introduces the scale, $s$, in the Sujatha PDF

$$
f_{s}(x ; b, s)=\frac{b^{3}\left(\frac{x^{2}}{s^{2}}+\frac{x}{s}+1\right) \mathrm{e}^{-\frac{b x}{s}}}{\left(b^{2}+b+2\right) s}
$$

which has the DF

$$
F_{s}(x ; b, s)=\frac{\left(\left(-s^{2}-s x-x^{2}\right) b^{2}-s(s+2 x) b-2 s^{2}\right) \mathrm{e}^{-\frac{b x}{s}}+s^{2}\left(b^{2}+b+2\right)}{s^{2}\left(b^{2}+b+2\right)} .
$$

We now determine some quantities, among others, its average value is

$$
\mu_{s}(b, s)=\mu(b) \times s,
$$

its variance is

$$
\sigma_{s}^{2}(b, s)=\sigma^{2}(b) \times s^{2},
$$




$$
\begin{aligned}
& \text { its median is at } \\
& \text { and its mode is } \\
& \qquad \operatorname{Med}_{s}(b, s)=\operatorname{Med}(b) \times s, \\
& \qquad \operatorname{Mode}_{s}(b, s)=\operatorname{Mode}(b) \times s . \\
& \text { The two parameters } b \text { and } s \text { can be derived by the numeri } \\
& \text { two following equations, which arise from the maximum like } \\
& \text { MLE), } \\
& \qquad \frac{3 n}{b}-\frac{n(2 b+1)}{b^{2}+b+2}-\frac{\sum_{i=1}^{n} x_{i}}{s}=0, \\
& \left.\qquad \frac{-3 n s+\left(\sum_{i=1}^{n} \frac{b x_{i}^{3}+b s x_{i}^{2}+s^{2}(b+1) x_{i}+2 s^{3}}{s^{2}+x_{i} s+x_{i}^{2}}\right.}{s^{2}}\right)
\end{aligned}
$$

The two parameters $b$ and $s$ can be derived by the numerical solution of the two following equations, which arise from the maximum likelihood estimator

where the $x_{i}$ are the elements of the experimental sample with $i$ varying between 1 and $n$.

\section{The Truncated Sujatha Distribution with Scale}

Let $X$ be a random variable with values in $\left[x_{l}, x_{u}\right]$. The truncated two-parameter Sujatha $\mathrm{PDF}, f_{T}(x)$, is

$$
f_{T}\left(x ; b, s, x_{l}, x_{u}\right)=\frac{b^{3}\left(s^{2}+s x+x^{2}\right) \mathrm{e}^{-\frac{b x}{s}}}{A},
$$

where

$$
\begin{aligned}
A= & \left(\left(s^{2}\left(b^{2}+b+2\right)+b x_{l}(b+2) s+b^{2} x_{l}^{2}\right) \mathrm{e}^{-\frac{x_{l} b}{s}}\right. \\
& \left.-\mathrm{e}^{-\frac{x_{u} b}{s}}\left(s^{2}\left(b^{2}+b+2\right)+b x_{u}(b+2) s+b^{2} x_{u}^{2}\right)\right) s .
\end{aligned}
$$

The truncated DF, $F_{T}$, is

$$
F_{T}\left(x ; b, s, x_{l}, x_{u}\right)=\frac{\left(\left(s^{2}+s x_{l}+x_{l}^{2}\right) b^{2}+s\left(s+2 x_{l}\right) b+2 s^{2}\right) \mathrm{e}^{-\frac{x_{l} b}{s}}-\left(\left(s^{2}+s x+x^{2}\right) b^{2}+s(s+2 x) b+2 s^{2}\right) \mathrm{e}^{-\frac{b x}{s}}}{B}
$$

where

$$
\begin{aligned}
B= & \left(\left(s^{2}+s x_{l}+x_{l}^{2}\right) b^{2}+s\left(s+2 x_{l}\right) b+2 s^{2}\right) \mathrm{e}^{-\frac{x_{l} b}{s}} \\
& -\left(\left(s^{2}+s x_{u}+x_{u}^{2}\right) b^{2}+s\left(s+2 x_{u}\right) b+2 s^{2}\right) \mathrm{e}^{-\frac{x_{u} b}{s}}
\end{aligned}
$$

Its average value, $\mu_{T}$, is

$$
\mu_{T}\left(b, s, x_{l}, x_{u}\right)=\frac{N}{D},
$$

where 


$$
\begin{aligned}
& N=\left(x_{l}\left(s^{2}+s x_{l}+x_{l}^{2}\right) b^{3}+s\left(s^{2}+2 s x_{l}+3 x_{l}^{2}\right) b^{2}+2 s^{2}\left(s+3 x_{l}\right) b+6 s^{3}\right) \mathrm{e}^{-\frac{x_{l} b}{s}} \\
& -\left(x_{u}\left(s^{2}+s x_{u}+x_{u}^{2}\right) b^{3}+s\left(s^{2}+2 s x_{u}+3 x_{u}^{2}\right) b^{2}+2 s^{2}\left(s+3 x_{u}\right) b+6 s^{3}\right) \mathrm{e}^{-\frac{x_{u} b}{s}}
\end{aligned}
$$

and

$$
\begin{aligned}
D= & b\left(\left(\left(s^{2}+s x_{l}+x_{l}^{2}\right) b^{2}+s\left(s+2 x_{l}\right) b+2 s^{2}\right) \mathrm{e}^{-\frac{x_{l} b}{s}}\right. \\
& \left.-\mathrm{e}^{-\frac{x_{u} b}{s}}\left(\left(s^{2}+s x_{u}+x_{u}^{2}\right) b^{2}+s\left(s+2 x_{u}\right) b+2 s^{2}\right)\right) .
\end{aligned}
$$

The first two parameters, $x_{l}$ and $x_{u}$, of the truncated Sujatha distribution can be obtained from the empirical data by evaluating the minimum and maximum elements of the sample. Consider a sample $\mathcal{X}=x_{1}, x_{2}, \cdots, x_{n}$ and let $x_{(1)} \geq x_{(2)} \geq \cdots \geq x_{(n)}$ denote their order statistics, so that $x_{(1)}=\max \left(x_{1}, x_{2}, \cdots, x_{n}\right)$, $x_{(n)}=\min \left(x_{1}, x_{2}, \cdots, x_{n}\right)$. The first two parameters $x_{l}$ and $x_{u}$ are

$$
x_{l}=x_{(n)}, \quad x_{u}=x_{(1)} .
$$

The remaining two parameters, $b$ and $s$, are obtained by maximizing the MLE but the results are complicated and therefore we omit them.

\section{The Luminosity Function}

The Sujatha luminosity function (LF) can be derived from Equation (13):

$$
\Psi\left(L ; b, L^{*}, \Psi^{*}\right) \mathrm{d} L=\Psi^{*} \frac{b^{3}\left(L^{2}+L L^{*}+L^{* 2}\right) \mathrm{e}^{-\frac{b L}{L^{*}}}}{\left(b^{2}+b+2\right) L^{* 3}},
$$

where $L$ is the luminosity, $L^{*}$ is the characteristic luminosity and $\Psi^{*}$ is a normalization, i.e. the number of galaxies per cubic Mpc. The mean luminosity per unit volume, $j$, is

$$
j=\int_{0}^{\infty} L \Psi(L) \mathrm{d} l=\frac{L^{*}\left(b^{2}+2 b+6\right) Y^{*}}{b\left(b^{2}+b+2\right)},
$$

and the average luminosity, $\langle L\rangle$, is

$$
\langle L\rangle=\frac{L^{*}\left(b^{2}+2 b+6\right)}{b\left(b^{2}+b+2\right)} .
$$

The version in absolute magnitude is

$$
\begin{aligned}
& \Psi\left(M ; b, M^{*}, \Psi^{*}\right) \mathrm{d} M \\
& =\frac{0.4 b^{3} Y^{*} \ln (10) \mathrm{e}^{-b 10^{-0.4 M+0.4 M^{*}}}\left(10^{-0.4 M+0.4 M^{*}}+10^{-1.2 M+1.2 M^{*}}+10^{-0.8 M+0.8 M^{*}}\right)}{b^{2}+b+2},
\end{aligned}
$$

where $M^{*}$ is the characteristic magnitude. We now introduce the parameter $h$, which is $H_{0} / 100$, where $H_{0}$ is the Hubble constant. The scaling with $h$ is $M^{*}-5 \log _{10} h$ and $\Psi^{*} h^{3}\left[\mathrm{Mpc}^{-3}\right]$. 


\section{The Truncated Luminosity Function}

We start with the truncated Sujatha PDF with scale as given by Equation (20)

$$
\Psi\left(L ; b, L^{*}, \Psi^{*}, L_{l}, L_{u}\right) \mathrm{d} L=\Psi^{*} \frac{b^{3}\left(L^{2}+L L^{*}+L^{* 2}\right) \mathrm{e}^{-\frac{b L}{L^{*}}} L^{*} \text { Fistar }}{E} \mathrm{~d} L,
$$

where $L$ is the luminosity, $L^{*}$ is the characteristic luminosity, $L_{l}$ is the lower boundary in luminosity, $L_{u}$ is the upper boundary in luminosity, $\Psi^{*}$ is a normalization factor and

$$
\begin{aligned}
E= & L^{* 2}\left(L^{* 2} \mathrm{e}^{-\frac{L_{l} b}{L^{*}}} b^{2}-L^{* 2} \mathrm{e}^{-\frac{L_{u} b}{L^{*}}} b^{2}+L^{*} \mathrm{e}^{-\frac{L_{l} b}{L^{*}}} b^{2} L_{l}-L^{*} \mathrm{e}^{-\frac{L_{u} b}{L^{*}}} b^{2} L_{u}+\mathrm{e}^{-\frac{L_{l} b}{L^{*}}} b^{2} L_{l}^{2}\right. \\
& -\mathrm{e}^{-\frac{L_{u} b}{L^{*}}} b^{2} L_{u}^{2}+L^{* 2} \mathrm{e}^{-\frac{L_{l} b}{L^{*}}} b-L^{* 2} \mathrm{e}^{-\frac{L_{u} b}{L^{*}}} b+2 L^{*} \mathrm{e}^{-\frac{L_{l} b}{L^{*}}} b L_{l}-2 L^{*} \mathrm{e}^{-\frac{L_{u} b}{L^{*}}} b L_{u} \\
& \left.+2 L^{* 2} \mathrm{e}^{-\frac{L_{l} b}{L^{*}}}-2 L^{* 2} \mathrm{e}^{-\frac{L_{u} b}{L^{*}}}\right) .
\end{aligned}
$$

The magnitude version is

$$
\Psi\left(M ; b, M^{*}, \Psi^{*}, M_{l}, M_{u}\right) \mathrm{d} L=\frac{F}{G},
$$

where

$$
\begin{aligned}
F= & -0.4 b^{3} \ln (10) \mathrm{e}^{-1.0 b 10^{-\frac{2 M}{5}+\frac{2 M^{*}}{5}}}\left(10^{0.4 M_{l}+0.4 M_{u}+0.4 M^{*}-1.2 M}\right. \\
& \left.+10^{0.4 M_{l}+0.4 M_{u}-0.8 M}+10^{0.4 M_{l}+0.4 M_{u}-0.4 M^{*}-0.4 M}\right),
\end{aligned}
$$

where

$$
\begin{aligned}
G= & \mathrm{e}^{-1.010^{-0.4 M_{l}+\frac{2 M^{*}}{5}} b} 10^{0.4 M_{l}+0.4 M_{u}-0.8 M^{*}} b^{2}+\mathrm{e}^{-1.010^{-0.4 M_{l}+\frac{2 M^{*}}{5}} b} 10^{0.4 M_{l}+0.4 M_{u}-0.8 M^{*}} b \\
& +2.0 \mathrm{e}^{-1.010^{-0.4 M_{l}+\frac{2 M^{*}}{5}} b} 10^{0.4 M_{l}+0.4 M_{u}-0.8 M^{*}}+\mathrm{e}^{-1.010^{-0.4 M_{l}+\frac{2 M^{*}}{5}} b} 10^{-0.4 M_{l}+0.4 M_{u}} b^{2} \\
& +\mathrm{e}^{-1.010^{-0.4 M_{l}+\frac{2 M^{*}}{5}} b} 10^{-0.4 M^{*}+0.4 M_{u}} b^{2}+2.0 \mathrm{e}^{-1.010^{-0.4 M_{l}+\frac{2 M^{*}}{5}} b} 10^{-0.4 M^{*}+0.4 M_{u}} b \\
& -\mathrm{e}^{-1.010^{-0.4 M_{u}+\frac{2 M^{*}}{5}} b} 10^{0.4 M_{l}+0.4 M_{u}-0.8 M^{*}} b^{2}-\mathrm{e}^{-1.010^{-0.4 M_{u}+\frac{2 M^{*}}{5}} b} 10^{0.4 M_{l}+0.4 M_{u}-0.8 M^{*}} b \\
& -2.0 \mathrm{e}^{-1.010^{-0.4 M_{u}+\frac{2 M^{*}}{5}} b} 10^{0.4 M_{l}+0.4 M_{u}-0.8 M^{*}}-\mathrm{e}^{-1.010^{-0.4 M_{u}+\frac{2 M^{*}}{5}}}{ }_{b} 10^{0.4 M_{l}-0.4 M_{u}} b^{2} \\
& -\mathrm{e}^{-1.010^{-0.4 M_{u}+\frac{2 M^{*}}{5}} b} 10^{-0.4 M^{*}+0.4 M_{l}} b^{2}-2.0 \mathrm{e}^{-1.010^{-0.4 M_{u}+\frac{2 M^{*}}{5}} b} 10^{-0.4 M^{*}+0.4 M_{l}} b,
\end{aligned}
$$

where $M$ is the absolute magnitude, $M^{*}$ the characteristic magnitude, $M_{l}$ the lower boundary in magnitude and $M_{u}$ the upper boundary in magnitude. The mean theoretical absolute magnitude, $\langle M\rangle$, is

$$
\langle M\rangle=\frac{\int_{M_{l}}^{M_{u}} M \times \Psi\left(M ; b, M^{*}, \Psi^{*}, M_{l}, M_{u}\right) \mathrm{d} M}{\int_{M_{l}}^{M_{u}} \Psi\left(M ; b, M^{*}, \Psi^{*}, M_{l}, M_{u}\right) \mathrm{d} M} .
$$




\section{The Photometric Maximum}

Following a procedure outlined in Section 5.4 of [7], the joint distribution in $Z$, the redshift, $f$, the flux and $\Omega$, the solid angle, for the number of galaxies in the case of the Sujatha distribution LF, see Equation (28), is

$$
\frac{\mathrm{d} N\left(z ; b, \Psi^{*}, z_{c r i t}\right)}{\mathrm{d} \Omega \mathrm{d} z \mathrm{~d} f}=\frac{4 z^{4} b^{3} \mathrm{e}^{-\frac{b z^{2}}{z_{\text {crit }}^{2}}} c_{l}^{5} \pi\left(z^{4}+z^{2} z_{c r i t}^{2}+z_{c r i t}^{4}\right)}{\left(b^{2}+b+2\right) H_{0}^{5} L^{*} z_{c r i t}^{4}},
$$

where the critical value of $z, z_{\text {crit }}$, is

$$
z_{c r i t}^{2}=\frac{H_{0}^{2} L^{*}}{4 \pi f c^{2}}
$$

where $L^{*}$ was defined in Section 5 and $c$ is the speed of light. Recall that in the pseudo-Euclidean universe, the flux of radiation, $f$, expressed in units of $\frac{L_{\odot}}{\mathrm{Mpc}^{2}}$ units, where $L_{\odot}$ represents the luminosity of the sun, is

$$
f=\frac{L}{4 \pi D^{2}}
$$

where $D$ is the distance of the galaxy expressed in Mpc, and

$$
D=\frac{c_{l} z}{H_{0}}
$$

The number of galaxies as a function of the redshift has a maximum at

$$
z=\frac{G}{6 \sqrt{b}\left(3 \sqrt{3} \sqrt{3 b^{4}+6 b^{3}-13 b^{2}+108 b+368} b+7 b^{3}+15 b^{2}+12 b+128\right)^{\frac{1}{6}}},
$$

where

$$
\begin{aligned}
G= & 2^{\frac{1}{6}} \sqrt{3}\left(-2 \times 2^{\frac{2}{3}}(b-4)\left(3 \sqrt{3} \sqrt{3 b^{4}+6 b^{3}-13 b^{2}+108 b+368} b+7 b^{3}+15 b^{2}+12 b+128\right)^{\frac{1}{3}}\right. \\
& \left.-8 b^{2}+2 \times 2^{\frac{1}{3}}\left(3 \sqrt{3} \sqrt{3 b^{4}+6 b^{3}-13 b^{2}+108 b+368 b}+7 b^{3}+15 b^{2}+12 b+128\right)^{\frac{2}{3}}+4 b+64\right)^{\frac{1}{2}} z_{c r i t} .
\end{aligned}
$$

Figure 2 shows the number of galaxies as a function of the parameter $b$ and the redshift $z$.

The mean redshift as a function of $z_{c r i t}$ and $b$ is

$$
\langle z\rangle\left(b, z_{\text {crit }}\right)=\frac{32 z_{\text {crit }}\left(b^{2}+3 b+12\right)}{3 \sqrt{\pi} \sqrt{b}\left(4 b^{2}+10 b+35\right)} .
$$

\section{Astrophysical Applications}

The merit function $\chi^{2}$, the reduced merit function $\chi_{\text {red }}^{2}$, the goodness of fit, $Q$, the Akaike information criterion (AIC), the maximum distance in the Kolmogorov-Smirnov test (K-S), $D$, and the significance level, $P_{K S}$, in the K-S test were defined in Section 5.1 of [7]. 


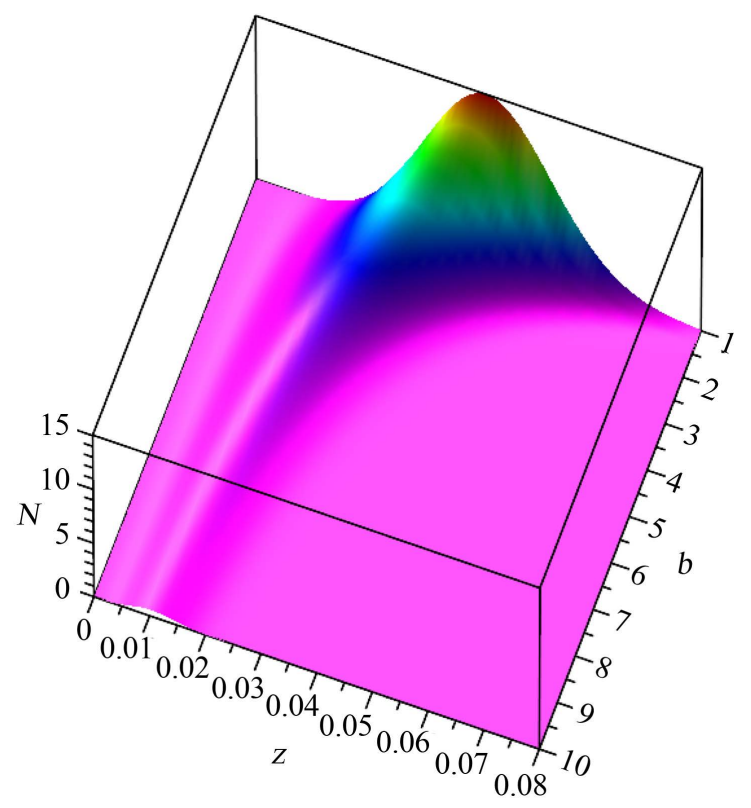

Figure 2. The number of galaxies as a function of the parameter $b$ and the redshift when $M^{*}=-23.7, f=1.29 \times 10^{6} \frac{L_{\odot}}{\mathrm{Mpc}^{2}}$ and $h=1$.

\subsection{The IMF}

We tested the truncated Sujatha distribution on four samples of stars: NGC 2362 (271 stars), the young cluster NGC 6611 (207 stars), the $\gamma$ Velorum cluster (237 stars), and the young cluster Berkeley 59 (420 stars). (For more details, see Section 5.2 of [8]). The results are presented in Table 1 for the truncated Sujatha distribution. The last column shows whether the results are better $(\mathrm{Y})$ than for the lognormal distribution or worse $(\mathrm{N})$. The results with the lognormal distribution were given in Table 1 in [8].

The empirical PDF visualized through histograms as well as the theoretical PDF for NGC 6611 are reported in Figure 3 and those for the $\gamma$ Velorum sample are reported in Figure 4.

\subsection{The Luminosity Function}

The first test was performed on the $u^{*}$ band of SDSS as in [9] with data available at https://cosmo.nyu.edu/blanton/lf.html. The Schechter function, the new Sujatha LF represented by Formula (28) and the data are reported in Figure 5 with parameters as in Table 2.

A careful examination of Table 2 reveals that the Sujatha LF has a bigger $\chi_{\text {red }}^{2}$ compared to the Schechter LF.

The second test is on the LF for quasi stellar objects (QSO) in the case $0.3<z<0.5$, see [10] for more details. Figure 6 displays the observed LF for QSO as well the theoretical fit with the Sujatha LF. The parameters and the statistical results for the Schechter LF are reported in Table 3 and those for the Sujatha LF in Table 4; the Sujatha LF has a bigger $\chi_{\text {red }}^{2}$ than the Schechter LF. 
Table 1. Numerical values of $\chi_{\text {red }}^{2}$, AIC, probability $Q, D$, the maximum distance between theoretical and observed DF, and $P_{K S}$, the significance level, in the K-S test of the truncated Sujatha distribution with two parameters for different mass distributions. The number of linear bins, $n$, is 20 .

\begin{tabular}{|c|c|c|c|c|c|c|c|}
\hline Cluster & parameters & AIC & $\chi_{\text {red }}^{2}$ & $Q$ & $D$ & $P_{K S}$ & $\mathrm{LN}$ \\
\hline NGC 2362 & $b=6.95 \times 10^{-2}, \quad s=1.61 \times 10^{-2}, \quad x_{l}=0.12, \quad x_{u}=1.47$ & 44.44 & 2.24 & $1.81 \times 10^{-3}$ & $4.85 \times 10^{-2}$ & 0.534 & $\mathrm{~N}$ \\
\hline NGC 6611 & $b=8.49, \quad s=3.57, \quad x_{l}=0.019, \quad x_{u}=1.46$ & 43.15 & 2.17 & $2.71 \times 10^{-3}$ & $5.67 \times 10^{-2}$ & 0.5 & $\mathrm{Y}$ \\
\hline$\gamma$ Velorum & $b=69297, \quad s=16663, \quad x_{l}=0.158, \quad x_{u}=1.317$ & 42.99 & 2.16 & $2.85 \times 10^{-3}$ & $6.14 \times 10^{-2}$ & 0.322 & $\mathrm{Y}$ \\
\hline Berkeley 59 & $b=3465, \quad s=1040, \quad x_{l}=0.16, \quad x_{u}=2.24$ & 78.12 & 4.11 & $8.96 \times 10^{-9}$ & 0.139 & $1.45 \times 10^{-7}$ & $\mathrm{~N}$ \\
\hline
\end{tabular}

Table 2. Numerical values and $\chi_{\text {red }}^{2}$ of the LFs applied to SDSS Galaxies in the $u^{*}$ band.

\begin{tabular}{ccc}
\hline LF & parameters & $\chi_{\text {red }}^{2}$ \\
\hline Schechter & $M^{*}=-17.92$, & $\alpha=-0.9, \quad \Phi^{*}=0.03 / \mathrm{Mpc}^{3}$ \\
Sujatha & $M^{*}=-20.70, \quad b=18.83, \quad \Psi^{*}=0.029 / \mathrm{Mpc}^{3}$ & 0.689 \\
\hline
\end{tabular}

Table 3. Parameters of the Schechter LF in the range of redshift $[0.3,0.5]$ when $k=3$ and $n=10$.

\begin{tabular}{ccccccc}
\hline$M^{*}$ & $\Psi^{*}$ & $\alpha$ & $\chi^{2}$ & $\chi_{\text {red }}^{2}$ & $Q$ & AIC \\
\hline-23.75 & $8.85 \times 10^{-7}$ & -1.37 & 10.491 & 1.49 & 0.162 & 16.49 \\
\hline
\end{tabular}

Table 4. Parameters of the Sujatha LF for QSOs in the range of redshift $[0.3,0.5]$ when $k=3$ and $n=10$.

\begin{tabular}{ccccccc}
\hline$M^{*}$ & $\Psi^{*}$ & $b$ & $\chi^{2}$ & $\chi_{\text {red }}^{2}$ & $Q$ & AIC \\
\hline-25.2 & $2.41 \times 10^{-6}$ & 12.56 & 32.05 & 4.57 & $3.96 \times 10^{-5}$ & 38.05 \\
\hline
\end{tabular}

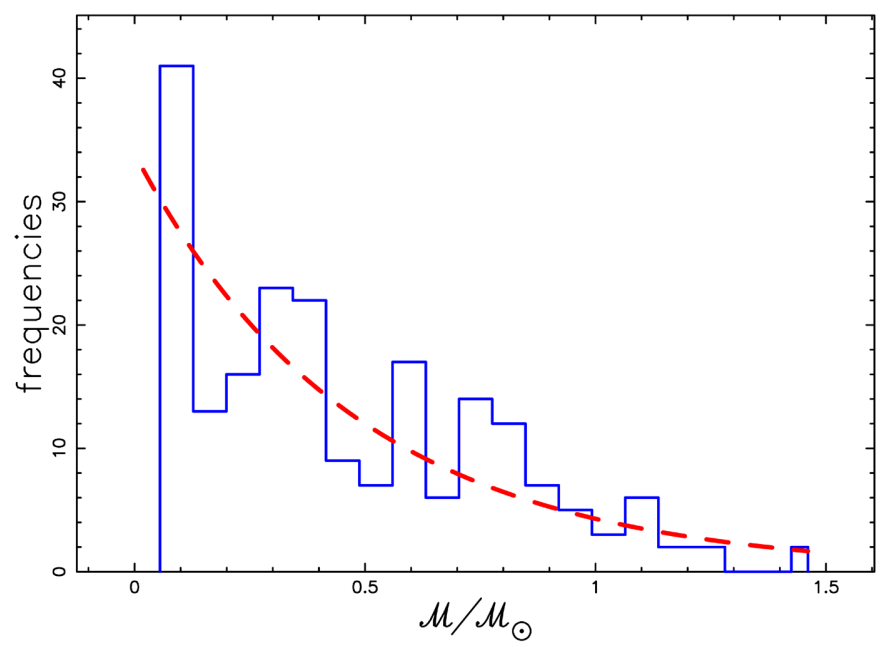

Figure 3. Empirical PDF of the mass distribution for NGC 6611 cluster data (blue histogram) with a superposition of the truncated Sujatha PDF (red line). Theoretical parameters as in Table 1. 


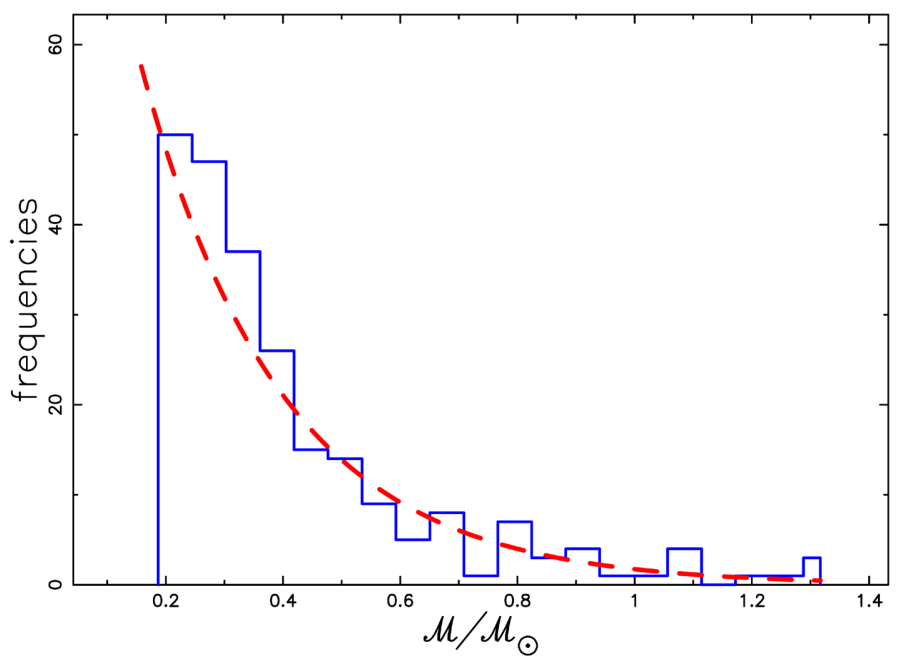

Figure 4. Empirical PDF of the mass distribution for $\gamma$ Velorum cluster data (blue histogram) with a superposition of the truncated Sujatha PDF (red line). Theoretical parameters as in Table 1.

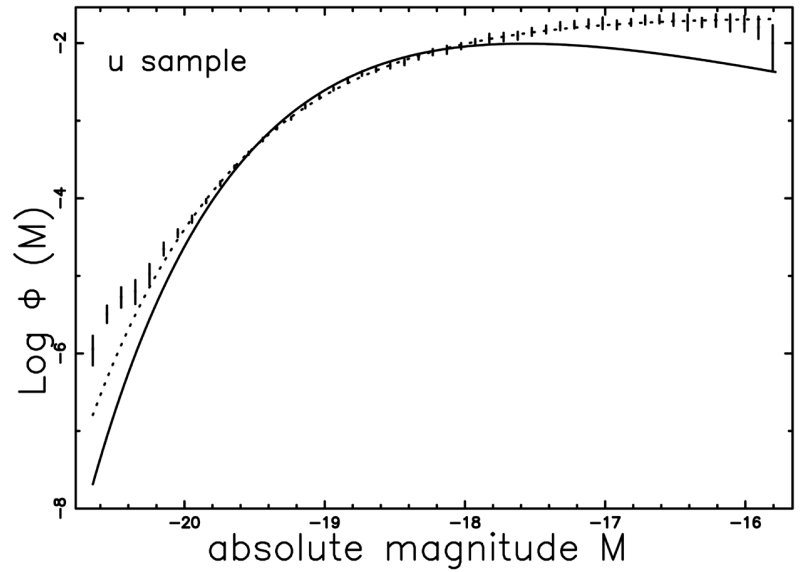

Figure 5. The LF data of SDSS $\left(u^{*}\right)$ are represented with error bars. The continuous line fit represents the Sujatha LF (28) and the dotted line represents the Schechter function.

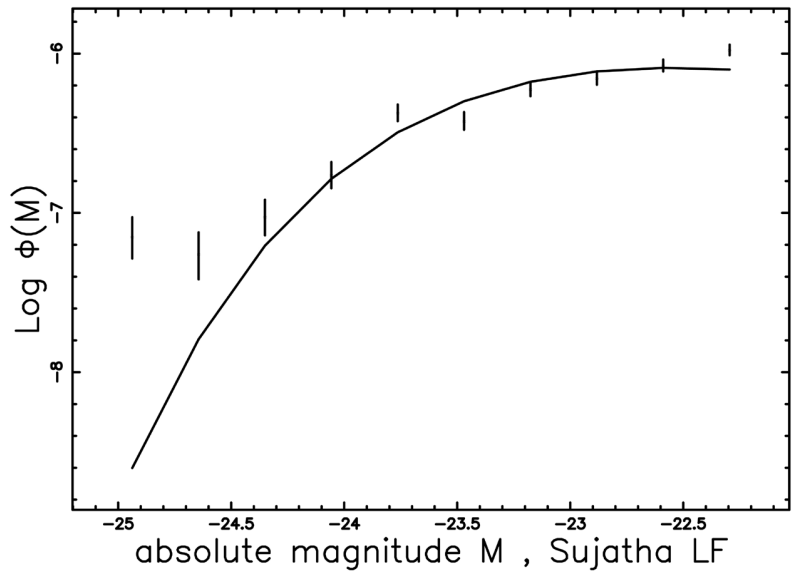

Figure 6. The observed LF for QSOs, empty stars with error bar, and the fit by the Sujatha LF for $z$ in $[0.3,0.5]$ and $M$ in $[-24.93,-22]$. Parameters as in Table 4. 


\subsection{The Photometric Maximum}

Figure 7 shows the number of observed galaxies for the 2MASS Redshift Survey (2MRS) catalog at a given apparent magnitude and both the Schechter and the Sujatha models for the number of galaxies as function of the redshift.

\subsection{Mean Absolute Magnitude}

The absolute magnitude that can be observed, as a function of the limiting apparent magnitude, $m_{L}$, is

$$
M_{L}=m_{L}-5 \log _{10}\left(\frac{c z}{H_{0}}\right)-25,
$$

where $m_{L}=11.75$ for the $2 \mathrm{MRS}$ catalog.

The theoretical average absolute magnitude of the truncated Sujatha LF, see Equation (37), can be compared with the observed average absolute magnitude of the 2MRS as a function of the redshift. To fit the data, we assumed the following empirical dependence on the redshift for the characteristic magnitude of the truncated Sujatha LF

$$
M^{*}=-25.14+2.5\left(1-\left(\frac{z-z_{\min }}{z_{\max }-z_{\min }}\right)^{0.7}\right) .
$$

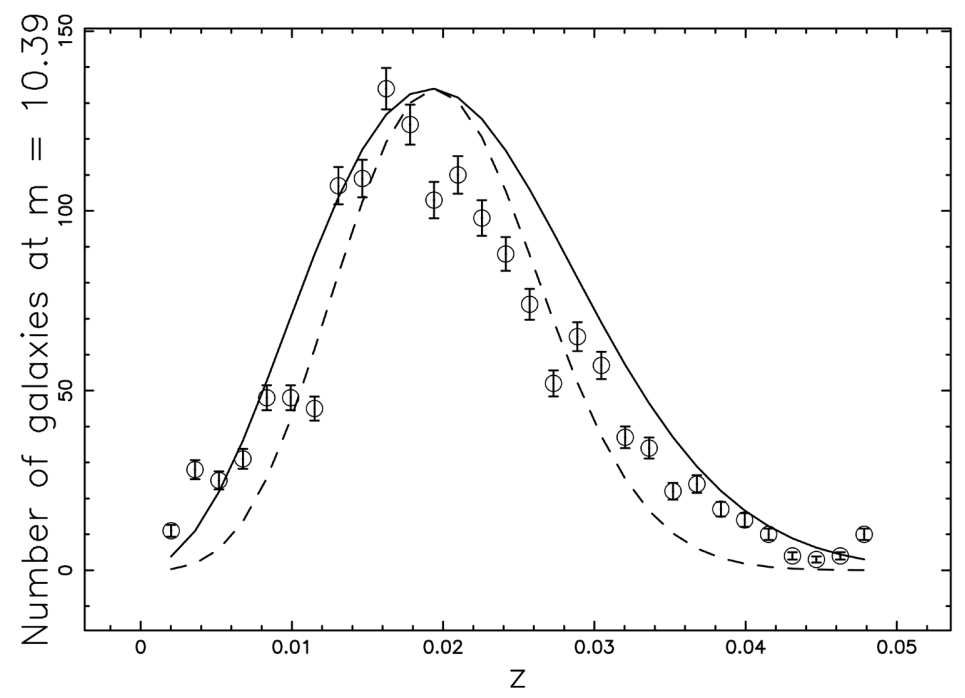

Figure 7. The galaxies of the $2 \mathrm{MRS}$ with $10.31 \leq m \leq 10.47$ or $1164793 \frac{L_{\odot}}{\mathrm{Mpc}^{2}} \leq f \leq 1346734 \frac{L_{\odot}}{\mathrm{Mpc}^{2}}$ are organized in frequencies versus heliocentric redshift, (empty circles); the error bar is given by the square root of the frequency. The maximum frequency of observed galaxies is at $z=0.017$. The full line is the theoretical curve generated by $\frac{\mathrm{d} N}{\mathrm{~d} \Omega \mathrm{d} z \mathrm{~d} f}(z)$ as given by the application of the Schechter LF which is Equation (43) in [11] and the dashed line represents the Sujatha LF which is Equation (38). The parameters for the Sujatha LF are $b=3.2$ and $M^{*}=-23.28, \chi^{2}=198.34$ for the Schechter LF and $\chi^{2}=205.18$ for the Sujatha LF. 


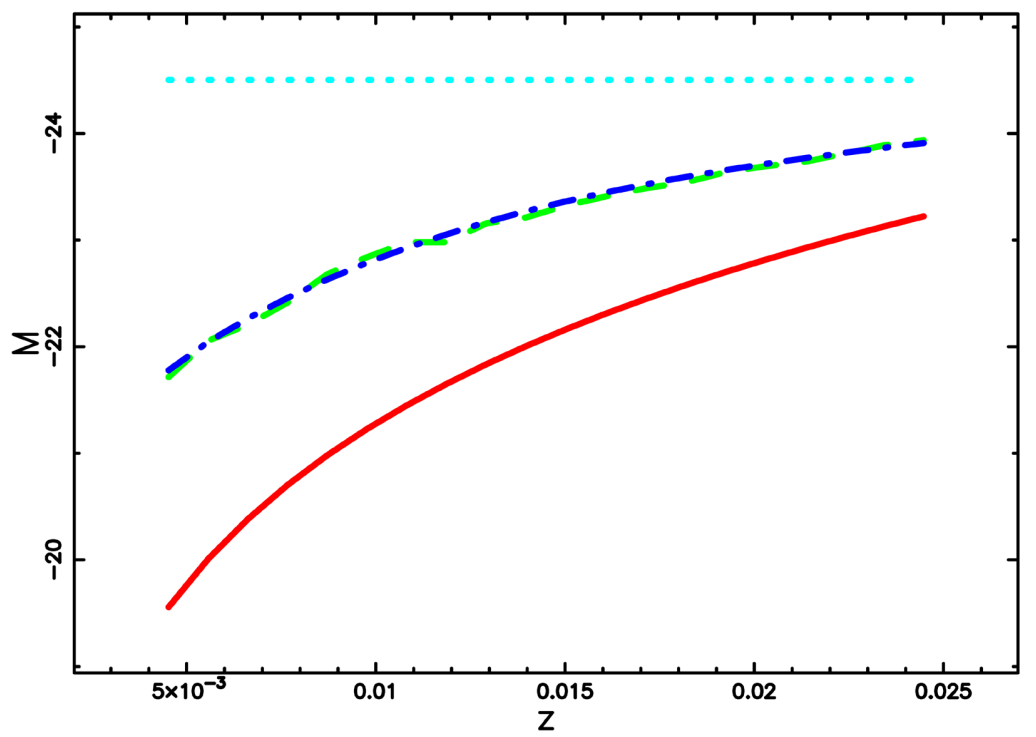

Figure 8. Average absolute magnitude of the galaxies belonging to the 2MRS (greendashed line), theoretical average absolute magnitude for the truncated Sujatha LF (blue dash-dot-dash-dot line) as given by Equation (37), lower theoretical curve as represented by Equation (45) (red line) and minimum absolute magnitude observed (cyan dotted line).

This relationship models the decrease of the characteristic absolute magnitude as a function of the redshift and allows us to match the observational and theoretical data. The lower bound in absolute magnitude is given by the minimum magnitude of the selected bin, the upper bound is given by Equation (45), the characteristic magnitude varies according to Equation (46) and Figure 8 shows a comparison between the theoretical and the observed absolute magnitude for the 2MRS catalog.

\section{Conclusions}

\section{Sujatha distribution with scale}

We derived the PDF, the DF, the average value, the variance, the median, the mode, an expression to generate random numbers and the way to obtain the two parameters, $b$ and $s$, by the MLE for the Sujatha distribution with scale.

\section{Truncated Sujatha distribution}

We derived the PDF, the DF, and the average value of the truncated Sujatha distribution. The application of this distribution to the IMF for stars gives better results than the lognormal distribution for two out of four samples, see Table 1.

\section{Sujatha luminosity function}

We derived the Sujatha LF in the standard and the truncated case. The application to both the SDSS Galaxies and to the QSOs in the range of redshift $[0.3,0.5]$ yields a lower reduced merit function compared to Schechter LF, see Table 2 and Table 4.

\section{Cosmological applications}

The number of galaxies as a function of the redshift, the flux and the solid an- 
gle for the Sujatha LF in the pseudo-Euclidean universe presents a maximum which can be compared with the observed one for the 2MRS, see Figure 7. The truncated Sujatha LF fits the average absolute magnitude of the 2MRS galaxies as a function of the redshift, see Figure 8, but the $\chi^{2}$ is bigger than that produced by the equivalent Schechter formula.

\section{Conflicts of Interest}

The author declares no conflicts of interest regarding the publication of this paper.

\section{References}

[1] Shanker, R. (2016) Sujatha Distribution and Its Applications. Statistics in Transition New Series, 17, 391-410. https://doi.org/10.21307/stattrans-2016-029

[2] Shanker, R. and Hagos, F. (2016) On Poisson-Sujatha Distribution and Its Applications to Model Count Data from Biological Sciences. Biometrics \& Biostatistics International Journal, 3, 100-106. https://doi.org/10.15406/bbij.2016.03.00069

[3] Shanker, R., Shukla, K.K. and Fesshaye, H. (2017) A Generalization of Sujatha Distribution and Its Applications with Real Lifetime Data. Journal of Institute of Science and Technology, 22, 66-83. https://doi.org/10.3126/jist.v22i1.17742

[4] Tesfay, M. and Shanker, R. (2018) A New-Two Parameter Sujatha Distribution with Properties and Applications. Türkiye Klinikleri Biyoistatistik, 10, 96-113. https://doi.org/10.5336/biostatic.2018-60954

[5] Tesfay, M. and Shanker, R. (2019) Another Two-Parameter Sujatha Distribution with Properties and Applications. Journal of Mathematical Sciences and Modelling, 2, 1-13. https://doi.org/10.33187/jmsm.416628

[6] Shanker, R., Shukla, K.K. and Leonida, T.A. (2020) A Two-Parameter Poisson-Sujatha Distribution. American Journal of Mathematics and Statistics, 68, 70-78.

[7] Zaninetti, L. (2021) New Probability Distributions in Astrophysics: V. The Truncated Weibull Distribution. International Journal of Astronomy and Astrophysics, 11, 133-149. https://doi.org/10.4236/ijaa.2021.111008

[8] Zaninetti, L. (2020) New Probability Distributions in Astrophysics: II. The Generalized and Double Truncated Lindley. International Journal of Astronomy and Astrophysics, 10, 39-55. https://doi.org/10.4236/ijaa.2020.101004

[9] Blanton, M.R., Hogg, D.W., Bahcall, N.A., Brinkmann, J. and Britton, M. (2003) The Galaxy Luminosity Function and Luminosity Density at Redshift $z=0.1$. The Astrophysical Journal, 592, 819-838. https://doi.org/10.1086/375776

[10] Zaninetti, L. (2017) A Left and Right Truncated Schechter Luminosity Function for Quasars. Galaxies, 5, 25. https://doi.org/10.3390/galaxies5020025

[11] Zaninetti, L. (2019) The Truncated Lindley Distribution with Applications in Astrophysics. Galaxies, 7, 61. https://doi.org/10.3390/galaxies7020061 